\title{
Kinematic Design of a Seven-Bar Linkage with Optimized Centrodes for Pure-Rolling Cutting
}

\author{
Biao Liu, ${ }^{1}$ Yali Ma, ${ }^{1}$ Delun Wang, ${ }^{1}$ Shaoping Bai, ${ }^{2}$ Yangyang Li, ${ }^{1}$ and Kangkang $\mathrm{Li}^{1}$ \\ ${ }^{1}$ School of Mechanical Engineering, Dalian University of Technology, Dalian, China \\ ${ }^{2}$ Department of Mechanical and Manufacturing Engineering, Aalborg University, Aalborg, Denmark \\ Correspondence should be addressed to Delun Wang; dlunwang@dlut.edu.cn
}

Received 9 February 2017; Revised 20 March 2017; Accepted 13 April 2017; Published 9 May 2017

Academic Editor: Anna M. Gil-Lafuente

Copyright (c) 2017 Biao Liu et al. This is an open access article distributed under the Creative Commons Attribution License, which permits unrestricted use, distribution, and reproduction in any medium, provided the original work is properly cited.

\begin{abstract}
A novel method for designing a seven-bar linkage based on the optimization of centrodes is presented in this paper. The proposed method is applied to the design of a pure-rolling cutting mechanism, wherein close interrelation between the contacting lines and centrodes of two pure-rolling bodies is formulated and the genetic optimization algorithm is adopted for the dimensional synthesis of the mechanism. The optimization is conducted to minimize the error between mechanism centrodes and the expected trajectories, subject to the design requirements of the opening distance, the maximum amount of overlap error, and peak value of shearing force. An optimal solution is obtained and the analysis results show that the horizontal slipping and standard deviation of the lowest moving points of the upper shear blade have been reduced by $78.0 \%$ and $80.1 \%$ and the peak value of shear stress decreases by $29 \%$, which indicate better cutting performance and long service life.
\end{abstract}

\section{Introduction}

A seven-bar linkage has two degrees of freedom, which can be used in many machines with variable trajectories. Of all associated machines, a typical example is the sevenbar pure-rolling cutting mechanism, which generates purerolling motion between two contacting bodies [1-4].

The design of pure-rolling mechanism is essentially a problem of trajectory synthesis of linkages, for which many synthesized methods are available. The synthesis can be carried out either for a set of given points or for a continuous trajectory $[5,6]$. The synthesis results are either exact or approximate. Normally exact synthesis is difficult to implement in practice and approximate methods are used to approximate the given points or continuous trajectory as much as possible [7]. To evaluate the trajectory deviation of approximate synthesis, some trajectory deviation measurement functions are introduced, including deterministic error [8], Fourier deviation [9], shape similarity [10], ambiguity function [11], and shape feature matching deviation [12].

Generally, there are two different ways to accomplish approximate synthesis, namely, direct and indirect synthesis methods. The direct synthesis method generates a mechanism directly according to the given points or continuous trajectory. Nelson Larsen [13] used an atlas of coupler curves to analyze the four-bar linkage, but the computation accuracy was unsatisfactory. Kramer [14] extended the selective precision synthesis method to generate four-bar motion mechanism with prescribed input crank rotations, which used the Hooke-and-Jeeves search method to handle the equality constraints during the synthesis process. Subbian and Flugrad [15] implemented the continuation method to deal with the sets of polynomial equations in the four-bar path generation synthesis, which was proved to be more effective. Nevertheless, even with these numerical methods, the nonlinear synthesis equations of high order are still difficult to solve. Cabrera et al. [8] used the genetic optimization algorithm to optimize the position error between the given target points and the points reached by the resulting mechanism during the synthesis of four-bar planar mechanisms. In order to obtain both effectiveness and high accuracy, many other optimization algorithms are also adopted in the trajectory synthesis of the mechanism, such as simulated annealing [16] and stochastic method [17]. 
The indirect synthesis method $[10,12]$ is used to search for the matching trajectory from the predefined trajectory atlas, instead of directly generating a mechanism scheme, which is done by analyzing the expected trajectory and then exporting the corresponding mechanism types and sizes. If there is a similar scheme, the minimum trajectory deviation will be obtained. The indirect synthesis method mainly relies on the mass data-storage capacity and rapid retrieval ability of a computer. Although the rapid improvement of computers promotes the application and development of the indirect synthesis, the difficulties of the establishment of a trajectory atlas, the mass data-storage capacity of a computer, and the approach to effectively search for the best matching trajectory are still challenging problems to be solved.

For the problem here, the design of seven-bar linkages for pure rolling needs to meet both the trajectory and also other requirements for machining, that is, steel plates cutting. The shear motion of a rolling shear mechanism is generally realized by means of the relative motion between the upper shear blade and the lower shear blade. The expected shear motion should be a pure-rolling motion without slipping. In this regard, Wang and Huang [18] developed an optimized model for rolling shear mechanism with single shaft and double eccentricity, choosing four motion positions as access points to acquire the expected motions, while the phase difference was set to be identical. Yang et al. [19] used the constraints of equal radius of crank and equal length of linkage to set up an optimization model of rolling shear mechanism with roll guide groove. Sun et al. [20] designed a rolling shear mechanism by optimizing the trajectory of the lowest moving point of the upper shear blade, but the upper shear blade could not perform pure-rolling motion relative to the lower blade due to the horizontal slide. In [21], a synthesis-optimized model was built to design a rolling shear mechanism, using a guiding rod as an additional design variable, while identical phase difference and identical length between the designed guide rod and the expected guide rod are adopted for four positions. In order to improve shear quality, decrease blade wear, and prolong blade life of the cutting machine [22], generally, the pure-rolling motion between the shear blades can be transformed into a series of moving positions and phase angles of the seven-bar linkages, with which an optimized method is adopted to obtain proper linkage sizes. In certain situations, the synthesis can only satisfy some key points; the motion accuracy of the designed pure-rolling cutting mechanism is thus low. It is difficult to realize the pure-rolling motion during the whole cutting process, due to the fact that the cutting performance was not considered or embodied in the synthesis.

This paper proposes a method for the kinematic design of a seven-bar linkage to generate pure-rolling motion by optimizing the centrodes. The introduced method is developed based upon the interrelation between the centrodes and contacting lines of pure-rolling motion. A case study of sevenbar rolling shear mechanism is included to demonstrate the method to accomplish the pure-rolling motion. A genetic optimization algorithm is used to obtain mechanism sizes with the metric function of minimum approximation error between mechanism centrodes and expected trajectories of

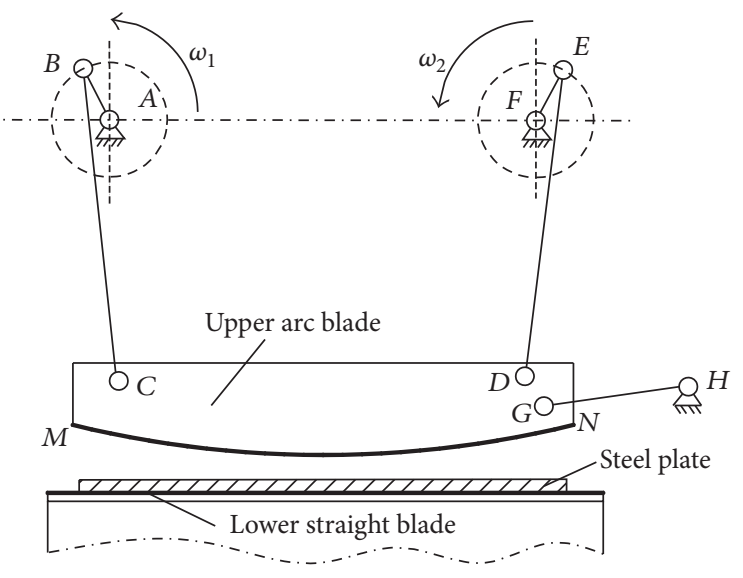

FIGURE 1: Sketch of a seven-bar rolling shear mechanism.

shear blade. The constraints of the formulated optimization problem for the pure-rolling mechanism include the design requirements of the opening distance, the maximum amount of overlap error, and peak value of shearing force. Moreover, the performance of the newly designed rolling shear mechanism is investigated and compared with the original one, which shows the advantages of the new method.

\section{Design Model}

2.1. Design Issue and Problem Formulation. The rolling shear mechanism is a typical pure-rolling cutting mechanism, which is usually used for medium plate rolling shear. The seven-bar linkage mechanism is a common application of the rolling shear mechanism [23], as shown in Figure 1.

The seven-bar linkage has 2 degrees of freedom, corresponding to cranks $A B$ and $E F$ as driving links, which rotate by the same angular velocity and with a constant phase difference, sharing a power input. Link CDG, to which the upper blade is attached, outputs motion. Generally, the lower shear blade is fixed on the frame, while the upper shear blade moves relative to the lower shear blade to cut the steel plate between them, as shown in Figure 2.

The horizontal sliding of the upper shear blade should be as little as possible to reduce the wear of the blade. Meanwhile, the cutting depth of the upper shear blade should be the same to reduce the bending deformation of the steel plate, ensuring a stable cutting quality of the steel plate. Thus, the ideal motion of the upper blade should be pure-rolling cutting relative to the steel plate during the shearing process, to make sure there is no horizontal sliding between the blade and the steel plate at the cutting contact point.

One of the rigid bodies is usually chosen to be fixed and another moves relative to the chosen one for convenience during the motion analysis of two rigid bodies, as shown in Figure 3. Rigid body II is fixed in coordinate system $\mathrm{O}_{f}-$ $x_{f} y_{f}$. Rigid body I, on which a moving coordinate system $O_{m}-x_{m} y_{m}$ is built, moves in the fixed coordinate system $O_{f}-x_{f} y_{f}$. A point $O_{m}$ of body I has a velocity $v_{O_{m}}$ and body I rotates about point $O_{m}$ with angular velocity $\omega_{O_{m}}$. The motion state of body I at any moment is either (a) entire translation 


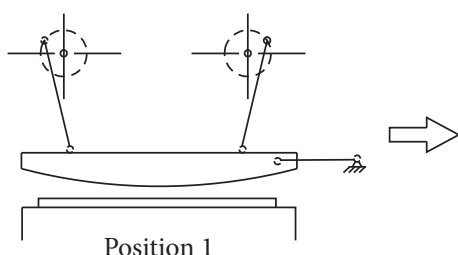

Position 1
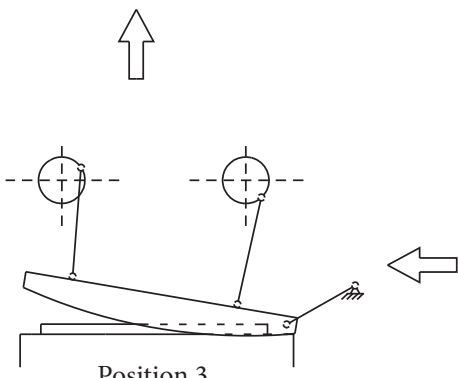

Position 3

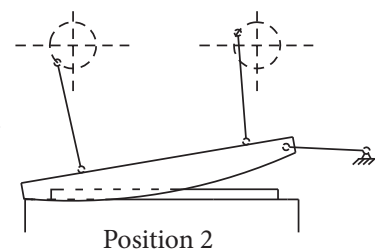

Position 2
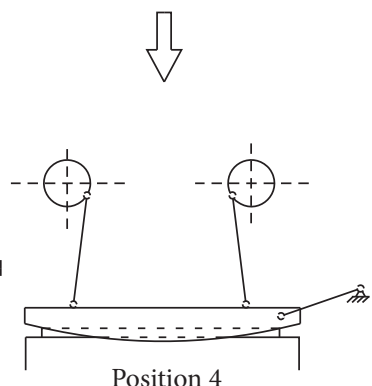

Position 4
FIgURE 2: Motion cycle of the rolling shear mechanism.

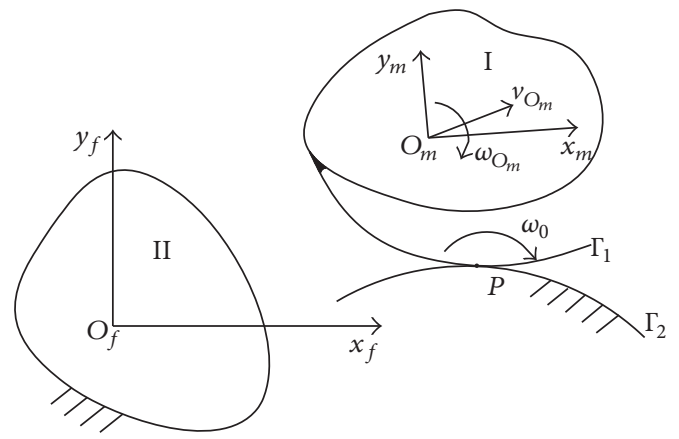

FIgURE 3: Fixed and moving centrodes of two rigid bodies.

or (b) rotation about a specific point $P$ on body I, of which the velocity in the fixed coordinate system is zero. The point $P$ is called the instantaneous velocity center, and the entire translation can be regarded as the point $P$ being at infinity. So, the motion of body I can be treated as a pure rotation about $P$ at any moment. As rigid body I moves, the instantaneous velocity center $P$ traces a trajectory in the fixed coordinate system $O_{f}-x_{f} y_{f}$, which is called the fixed centrode $\Gamma_{1}$, and a trajectory in the moving coordinate system $O_{m}-x_{m} y_{m}$, which is called the moving centrode $\Gamma_{2}$. The motion of body I can be regarded as the pure-rolling motion of the moving centrode along with the fixed centrode with no sliding.

As the thickness of the steel plate is far less than the width of the steel plate and the length of the blade, the contact line of the shear blade and steel plate is usually treated as a contact point in practice. Thus, the ideal cutting motion can be regarded as the pure-rolling motion between the upper shear blade and the lower shear blade with no sliding at the contact point. The objective is to synthesize the linkage for pure-rolling shear motion, so that the profiles of the upper and lower blades coincide with the moving and fixed centrodes of the output link $C D G$, respectively, during the shearing process.
2.2. Kinematic Design Model. A seven-bar linkage is chosen to establish the kinematic design model, of which the topological structure can be obtained, as shown in Figure 4 .

Links $A B$ and $E F$ are assigned as the driving links, while the ternary link $C D G$ is assigned as the output coupler, to which the upper shear blade is attached. Link $A F H$, which is also a ternary link assigned number 7 , is chosen as the frame. So, there are 6 movable links, corresponding to 6 angles $\left\{\theta_{i}, i=1,2, \ldots, 6\right\}$, as shown in Figure 5. Vector equations of closed-loop HGDEFH and HGCBAH are listed as follows:

$$
\begin{aligned}
& \mathrm{HG}+\mathrm{GD}+\mathrm{DE}=\mathrm{HF}+\mathrm{FE}, \\
& \mathrm{HG}+\mathrm{GC}+\mathrm{CB}=\mathrm{HA}+\mathrm{AB} .
\end{aligned}
$$

Usually, joints $A$ and $F$ are designed with the same height for convenience of structure design and power transmission. For the convenience of the modeling, vectors $\mathbf{H K}$ and $\mathbf{K F}$ are introduced to take the place of vectors $\mathbf{H A}$ and $\mathbf{H F}$, as shown in Figure 5. The lengths of vectors $\mathbf{H K}$ and $\mathrm{KF}$ represent the vertical and horizontal distances of joint $F$ relative to joint $H$, respectively.

So, (2) can be obtained from the new closed-loop HGDEFKH and HGCBAFKH as

$$
\begin{aligned}
& \mathrm{HG}+\mathrm{GD}+\mathrm{DE}=\mathrm{HK}+\mathrm{KF}+\mathrm{FE} \\
& \mathrm{HG}+\mathrm{GC}+\mathrm{CB}=\mathrm{HK}+\mathrm{KF}+\mathrm{FA}+\mathrm{AB} .
\end{aligned}
$$

A fixed coordinate system $H-x y$ and a moving coordinate system $L-x_{m} y_{m}$ are established at the hinged point $H$ and the center of the driven link $C D$, respectively. Besides the basic length parameters $\left\{L_{i}, i=1,2, \ldots, 6\right\}$ of the 6 movable links shown in Figure $5, L_{7}, L_{8}$, and $\alpha$ are introduced to determine the dimensions of link $C D G$, and $L_{9}, L_{10}$, and $L_{11}$ are introduced for vectors $\mathbf{A F}, \mathbf{H K}$, and $\mathbf{K F}$. Thus, the length parameters of the linkage are $\left\{L_{i}, i=1,2, \ldots, 11\right\}$. Expanding (2) yields

$$
\begin{aligned}
& L_{6} \cos \theta_{6}+L_{5} \cos \theta_{5}+L_{4} \cos \theta_{4}+L_{11} \\
& \quad-L_{2} \cos \theta_{2}=0, \\
& L_{6} \sin \theta_{6}+L_{5} \sin \theta_{5}+L_{4} \sin \theta_{4}-L_{10}-L_{2} \sin \theta_{2}=0, \\
& L_{6} \cos \theta_{6}+L_{8} \cos \left(\theta_{5}+\alpha\right)+L_{3} \cos \theta_{3}+L_{9}+L_{11} \\
& \quad-L_{1} \cos \theta_{1}=0, \\
& L_{6} \sin \theta_{6}+L_{8} \sin \left(\theta_{5}+\alpha\right)+L_{3} \sin \theta_{3}-L_{10} \\
& \quad-L_{1} \sin \theta_{1}=0 .
\end{aligned}
$$

The driving links, $A B$ and $E F$, have the same angular velocity with a constant phase difference, sharing a power 


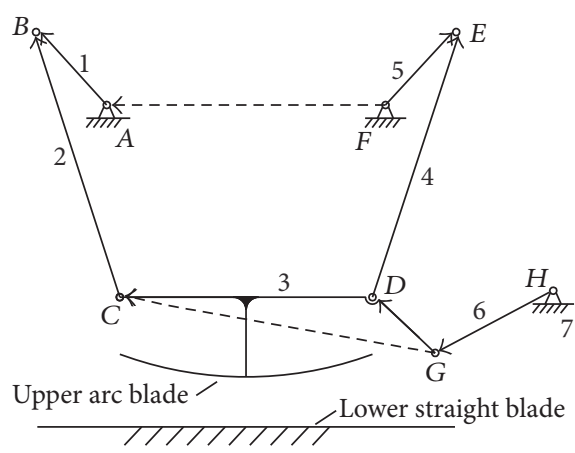

(a) Schematic diagram of the mechanism

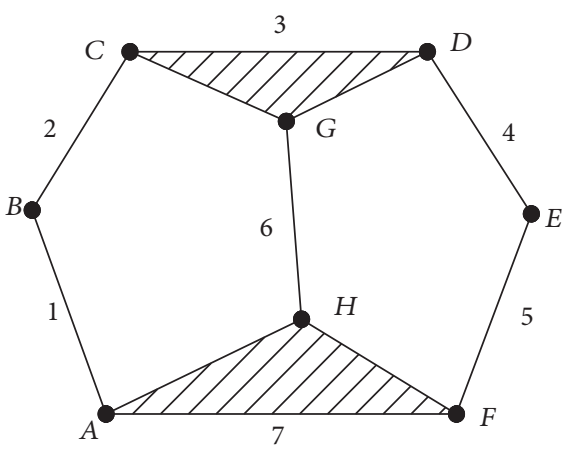

(b) Topological structure of the mechanism

FIGURE 4: Schematic diagram and topological structure of the seven-bar linkage.

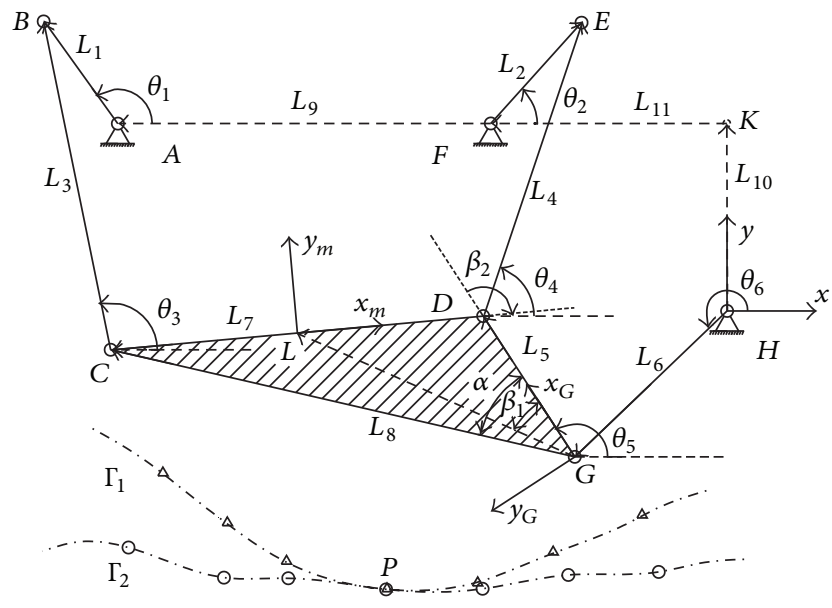

FIGURE 5: Kinematic model of the seven-bar linkage.

input. That means $\theta_{1}-\theta_{2}=\theta_{C}$. The differentiation with respect to time of (3) yields

$$
\begin{aligned}
& -L_{6} \omega_{6} \sin \theta_{6}-L_{5} \omega_{5} \sin \theta_{5}-L_{4} \omega_{4} \sin \theta_{4} \\
& \quad+L_{2} \omega_{2} \sin \theta_{2}=0, \\
& L_{6} \omega_{6} \cos \theta_{6}+L_{5} \omega_{5} \cos \theta_{5}+L_{4} \omega_{4} \cos \theta_{4} \\
& \quad-L_{2} \omega_{2} \cos \theta_{2}=0, \\
& -L_{6} \omega_{6} \sin \theta_{6}-L_{8} \omega_{5} \sin \left(\theta_{5}+\alpha\right)-L_{3} \omega_{3} \sin \theta_{3} \\
& \quad+L_{1} \omega_{1} \sin \theta_{1}=0, \\
& L_{6} \omega_{6} \cos \theta_{6}+L_{8} \omega_{5} \cos \left(\theta_{5}+\alpha\right)+L_{3} \omega_{3} \cos \theta_{3} \\
& \quad-L_{1} \omega_{1} \cos \theta_{1}=0,
\end{aligned}
$$

where $\omega_{1}=\omega_{2}$, which are given quantities, denoting the angular velocity of links $A B$ and $E F$. Thus, $\theta_{5}, \theta_{6}, \omega_{5}$, and $\omega_{6}$ can be obtained by solving (3) to (4).

The driven link CDG should generate pure-rolling cutting motion between the upper and lower blades. During the cutting process, the instantaneous center $P$ forms the moving centrode relative to the driven link $C D G$ and the fixed centrode relative to the fixed frame, represented by curves $\Gamma_{1}$ and $\Gamma_{2}$, as displayed in Figure 5. In order to derive the kinematic equations of centrodes, coordinate transformation matrix is used to transform the points from moving coordinate system to fixed coordinate system, which is related to rotation angle and translation distance [24]. Let the coordinates of instantaneous center $P$ be $(x, y)$ in the fixed coordinate system $H-x y$ and $\left(x_{m}, y_{m}\right)$ in the moving coordinate system $L-x_{m} y_{m}$. An additional coordinate system $G-x_{G} y_{G}$, which is established at hinged point $G$ as shown in Figure 5, is introduced to implement coordinate transform between the fixed and moving coordinate systems. The two sets of coordinates $(x, y)$ and $\left(x_{m}, y_{m}\right)$ are related by

$$
\left\{\begin{array}{l}
x \\
y \\
1
\end{array}\right\}=\mathbf{M}_{H G} \mathbf{M}_{G L}\left\{\begin{array}{c}
x_{m} \\
y_{m} \\
1
\end{array}\right\},
$$

where matrix $\mathbf{M}_{H G}$ is the homogeneous transformation matrix from the fixed coordinate system $H-x y$ to $G-x_{p} y_{p}$ and $\mathbf{M}_{G L}$ is the one from $G-x_{p} y_{p}$ to the moving coordinate system $L-x_{m} y_{m}$. They are given as

$$
\begin{aligned}
& \mathbf{M}_{H G}=\left[\begin{array}{ccc}
\cos \theta_{5} & -\sin \theta_{5} & L_{6} \cos \theta_{6} \\
\sin \theta_{5} & \cos \theta_{5} & L_{6} \sin \theta_{6} \\
0 & 0 & 1
\end{array}\right], \\
& \mathbf{M}_{G L}=\left[\begin{array}{ccc}
\cos \beta_{2} & \sin \beta_{2} & L_{G L} \cos \beta_{1} \\
-\sin \beta_{2} & \cos \beta_{2} & L_{G L} \sin \beta_{1} \\
0 & 0 & 1
\end{array}\right],
\end{aligned}
$$

where $\theta_{5}$ and $\theta_{6}$ are orientation angles of links $D G$ and $G H$, $\beta_{1}$ is the angle between vectors GD and GL, and $\beta_{2}$ is the orientation angle of the $x$-axis of $L-x_{m} y_{m}$ in coordinate system $G-x_{G} y_{G} ; L_{G L}$ represents the length of GL. Substituting the above equation into (5) and upon differentiation with respect to time, one has 


$$
\left\{\begin{array}{l}
\dot{x} \\
\dot{y} \\
0
\end{array}\right\}=\left[\begin{array}{ccc}
-\omega_{5} \sin \theta_{5} & -\omega_{5} \cos \theta_{5} & -L_{6} \omega_{6} \sin \theta_{6} \\
\omega_{5} \cos \theta_{5} & -\omega_{5} \sin \theta_{5} & L_{6} \omega_{6} \cos \theta_{6} \\
0 & 0 & 0
\end{array}\right]\left[\begin{array}{ccc}
\cos \beta_{2} & \sin \beta_{2} & L_{G L} \cos \beta_{1} \\
-\sin \beta_{2} & \cos \beta_{2} & L_{G L} \sin \beta_{1} \\
0 & 0 & 1
\end{array}\right]\left\{\begin{array}{c}
x_{m} \\
y_{m} \\
1
\end{array}\right\},
$$

where $\dot{x}$ and $\dot{y}$ are the velocities of instantaneous center $P . \omega_{5}$ and $\omega_{6}$ are the angular velocities of links $L_{5}$ and $L_{6}$. As the velocity of instantaneous velocity center $P$ at any moment in the fixed coordinate system $H-x y$ is zero, namely, $\dot{x}=\dot{y}=0$, by arranging and rewriting the above equation, the moving centrode of link $C D G$ is expressed as

$$
\begin{aligned}
& x_{m}=L_{G L} \omega_{5} \cos \left(\beta_{1}+\beta_{2}\right)+L_{6} \omega_{6} \cos \left(\beta_{2}+\theta_{6}-\theta_{5}\right), \\
& y_{m}=L_{G L} \omega_{5} \sin \left(\beta_{1}+\beta_{2}\right)+L_{6} \omega_{6} \sin \left(\beta_{2}+\theta_{6}-\theta_{5}\right) .
\end{aligned}
$$

Substituting (8) into (5), the fixed centrode is obtained as

$$
\begin{aligned}
& x=L_{G L}\left(\omega_{5}+1\right) \cos \left(\beta_{1}+\theta_{5}\right)+L_{6}\left(\omega_{6}+1\right) \cos \theta_{6}, \\
& y=L_{G L}\left(\omega_{5}+1\right) \sin \left(\beta_{1}+\theta_{5}\right)+L_{6}\left(\omega_{6}+1\right) \sin \theta_{6} .
\end{aligned}
$$

So far, both the moving and the fixed centrodes have been obtained, upon which optimal sizes and positions of the mechanism can be searched to ensure that the trajectories of moving and fixed centrodes cooperate with each other in the way of pure rolling.

\section{Optimization Design Case}

In this section, a design case of seven-bar rolling shear mechanism, as a kind of common pure-rolling cutting mechanism, is considered. The ideal shear motion of a rolling shear mechanism should be pure-rolling motion between the upper shear blade and the lower shear blade. With the generated moving centrodes and fixed centrodes coinciding with the motion contact lines of the upper shear blade and lower shear blade, respectively, the pure-rolling motion can be obtained. Hence, the optimization objective function and the constraints could be determined by pure-rolling motion and cutting performance requirements. A genetic optimization method [25] is employed to determine the proper linkage sizes of rolling shear mechanism thanks to its effectiveness and convenience.

3.1. Design Parameters. The design parameters of a rolling shear mechanism are given by the cutting process [26, 27]. These design parameters include the width of sheared plate $B$, the maximum shearing thickness $h_{\max }$, the shearing overlap $S$, and the shearing angle $\alpha$, as shown in Figure 6 . The width $B$ determines the horizontal width of the lower shear blade, and the shearing overlap $S$ gives the overlapping amount between the upper and the lower shear blades in the shearing process. The shear angle $\alpha$ refers to the contact point between the lower shear blade and the tangent of arc upper shear blade.
3.2. Optimization Model. Based on the design parameters, the expected trajectories (or profiles) of upper and lower shear blades can be obtained. The purpose of genetic optimization model is to seek a set of optimal mechanism sizes to minimize the deviation between centrodes and expected trajectories of upper and lower shear blades, subject to some specific design requirements. The detailed optimization model is as follows.

3.2.1. Optimization Variables. The design variables of a rolling shear mechanism are generally the lengths of links and pivoting joint positions. These design variables are defined as optimization variables, expressed by a vector $\mathbf{t}$ :

$$
\mathbf{t}=\left[t_{1}, t_{2}, \ldots, t_{n}\right]^{T}, \quad \mathbf{t} \in R^{n},
$$

in which each variable $t_{i}(i=1,2, \ldots, n)$ represents the size parameter of a mechanism scheme, such as the lengths of links $\left\{L_{i}, i=1,2, \ldots, 11\right\}$ and phase angles $\left\{\theta_{i}, i=\right.$ $1,2, \ldots, 6\}$. Each optimal scheme can be expressed by vector $\mathbf{t}^{*}$, called optimal point.

3.2.2. Optimization Objective Function. The objective of the design optimization is to make the moving centrode approach the profile of the upper shear blade and the fixed centrode approach the profile of the lower shear blade as much as possible. Accordingly, the objective function of the optimization design can be defined as the sum of approaching errors, including the approaching error for moving centrode and upper shear blade, together with the approaching error for fixed centrode and lower shear blade, which will be minimized as follows:

$$
\begin{array}{ll}
\min & U(\mathbf{t}) \\
& U(\mathbf{t})=U_{1}(\mathbf{t})+U_{2}(\mathbf{t}),
\end{array}
$$

where $U_{1}(\mathbf{t})$ and $U_{2}(\mathbf{t})$ are the curve approximation errors between moving centrode and upper shear blade and fixed centrode and lower shear blade, respectively. The errors should be evaluated in the moving coordinate system $O_{m}-$ $x_{m} y_{m}$ and the fixed coordinate system $O-x y$ on upper and lower shear blade, as shown in Figure 7.

The geometric equations of the moving centrode and profile of the upper shear blade in the moving coordinate system $O_{m}-x_{m} y_{m}$ can be written as

$$
\begin{aligned}
& y_{m}=g_{m t}\left(x_{m}\right), \\
& y_{m}=f_{m}\left(x_{m}\right)\left(x_{m 1} \leq x_{m} \leq x_{m n}\right) .
\end{aligned}
$$




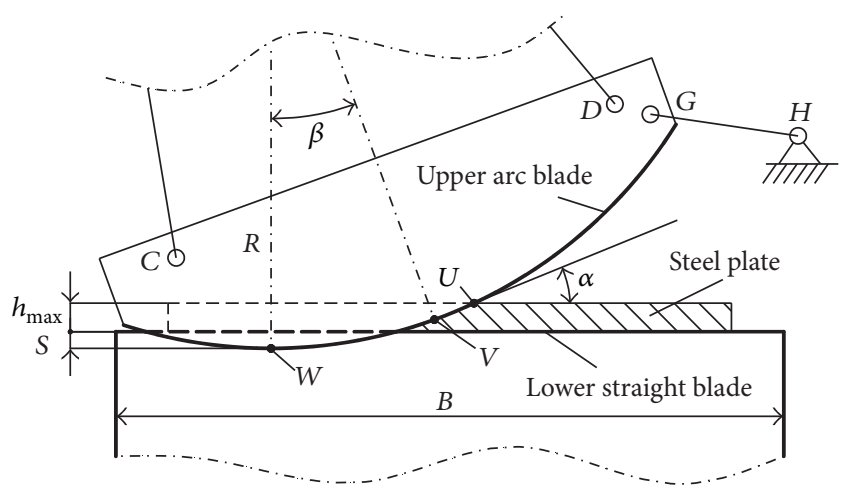

FIGURE 6: Design parameters of the rolling shear mechanism.

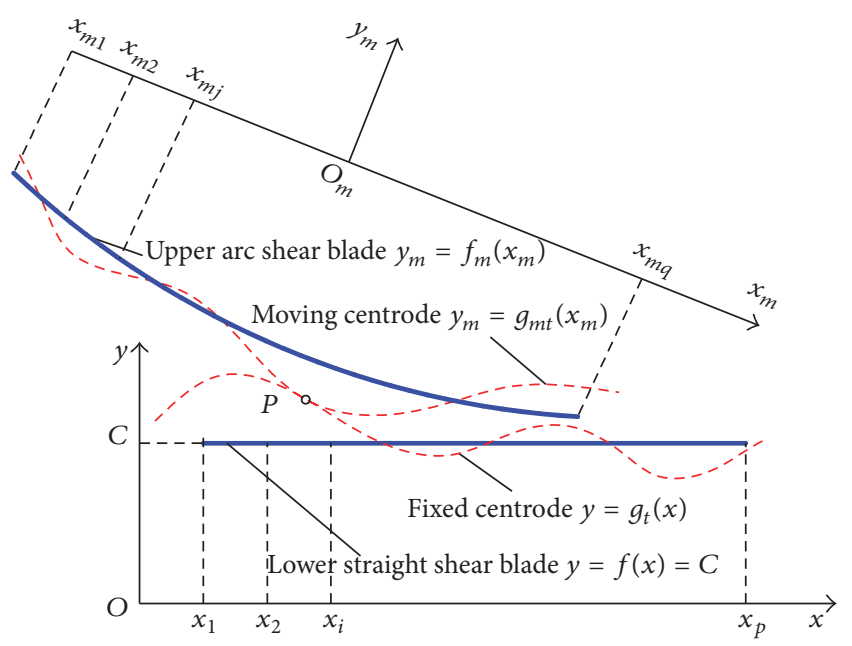

FIgURE 7: Centrodes and profiles of the shear blades.

Also, equations of the fixed centrode and profile of the lower shear blade in the fixed coordinate system $O-x y$ can be written as

$$
\begin{aligned}
& y=g_{t}(x), \\
& y=C \quad\left(x_{1} \leq x \leq x_{n}\right),
\end{aligned}
$$

where $x_{n}-x_{1}=B$ and $C$ is a constant, describing the position of the sheared plate.

The errors $U_{1}(\mathbf{t})$ and $U_{2}(\mathbf{t})$ can be determined by

$$
\begin{aligned}
& U_{1}(\mathbf{t})=\sqrt{\frac{1}{n} \sum_{i=1}^{n}\left(g_{t}\left(x_{i}\right)-C\right)^{2},} \\
& U_{2}(\mathbf{t})=\sqrt{\frac{1}{n} \sum_{i=1}^{n}\left(g_{m t}\left(x_{m i}\right)-f_{m}\left(x_{m i}\right)\right)^{2}} .
\end{aligned}
$$

Hence, the objective function of optimization for the pure-rolling cutting mechanism design can be expressed as

$$
\begin{aligned}
U(\mathbf{t})= & \sqrt{\frac{1}{n} \sum_{i=1}^{n}\left(g_{t}\left(x_{i}\right)-f\left(x_{i}\right)\right)^{2}} \\
& +\sqrt{\frac{1}{n} \sum_{i=1}^{n}\left(g_{m t}\left(x_{m i}\right)-f_{m}\left(x_{m i}\right)\right)^{2}} .
\end{aligned}
$$

3.2.3. Constraints. The constraints of a rolling shear mechanism mainly include some motion parameters and performance parameters, such as the opening distance, the shearing overlap error of the upper and lower shear blade, and the peak value of shearing force.

(1) Opening Distance Constraint. In order to make the sheared plate get through smoothly between the two shear blades, the clearance between the upper and lower shear blades after shearing, also known as the opening distance $(H)$, which is the function of design variable $t$, should be greater than the designed value $K$ associated with the thickness of sheared plate:

$$
H(\mathbf{t}) \geq K
$$

(2) Overlap Error Constraint. The overlap error in direction of plate width should be limited to a given amount. The overlap amount is the distance from the lowest moving point of upper shear blade to the lower shear blade. The coordinates of the lowest moving point $W$ in the fixed coordinate system can be obtained by a geometrical relationship as shown in Figure 6. It can be written as

$$
\begin{aligned}
& x_{W}=x_{V}-R \sin \beta, \\
& y_{W}=y_{V}-R(1-\cos \beta),
\end{aligned}
$$

where $R$ and $\beta$ are the arc radius and the dip angle of upper shear blade, respectively, and $\left(x_{V}, y_{V}\right)$ is the coordinate of middle point $V$ on upper shear blade in the fixed coordinate system. Thus, the overlap error constraint is expressed as

$$
|\Delta S|=\left|C-y_{W}-S\right| \leq 0.1 \cdot S .
$$

(3) Peak Value of Shearing Force Constraint. Generally, the forces applied on upper shear blade refer to both shear force and other forces, such as friction force. The peak value of shear force constraint can be introduced by limiting the maximum shearing force that usually appears in the initial shear stage. The shearing force [28] of a rolling shear mechanism is expressed as

$$
\begin{aligned}
P= & 0.6 \sigma_{b} \delta \\
& \cdot \frac{h^{2}}{\tan \alpha}\left(1+Z \frac{\tan \alpha}{0.6 \delta}+\frac{1}{1+100 \delta / \sigma_{b} Y^{2} X}\right),
\end{aligned}
$$


TABLE 1: Design parameters of the rolling shear mechanism.

\begin{tabular}{lcccc}
\hline $\begin{array}{l}\text { Plate } \\
\text { width } B\end{array}$ & $\begin{array}{c}\text { Maximum } \\
\text { thickness } \\
h_{\max }\end{array}$ & $\begin{array}{c}\text { Overlap } \\
\text { amount } S\end{array}$ & $\begin{array}{c}\text { Shear } \\
\text { angle } \alpha\end{array}$ & $\begin{array}{c}\text { Opening } \\
\text { distance } H\end{array}$ \\
\hline $3500 \mathrm{~mm}$ & $50 \mathrm{~mm}$ & $5 \mathrm{~mm}$ & $2.24^{\circ}$ & $\geq 200 \mathrm{~mm}$ \\
\hline
\end{tabular}

where $\sigma_{B}$ and $\delta$ are the ultimate strength and percentage elongation of material for sheared plate, $Z$ represents the conversion coefficient, $Y$ is the ratio of shear blade gap with the thickness of steel plate, and $X$ is the ratio of the distance between shear blade edge and steel plate with the thickness of steel plate. The shearing force constraint may be limited by shear angle $\alpha$, because the peak value of shearing force can be highly correlated to the shear angle. Therefore, it may be given by means of specified shear angle $\alpha_{0}$, which is written as

$$
\alpha_{\mathrm{st}} \geq \alpha_{0}
$$

where $\alpha_{\text {st }}$ is the initial shear angle of upper shear blade. According to the above discussion for determining the sizes of rolling shear mechanism, the final design vector, marked as $\mathbf{t}^{*}$, where the mechanism sizes achieve pure-rolling motion of the upper shear blade, can be obtained by means of the genetic optimization algorithm.

\section{Results and Analysis}

According to the optimization functions and given shearing requirements, the seven-bar mechanism for pure-rolling cutting will be synthesized and the kinematic performance will be analyzed and compared to the original one.

4.1. Optimization Results. The seven-bar mechanism for pure-rolling cutting is shown in Figure 1. The actual design parameters of sheared plate are used as the design parameters of rolling shear mechanism, as shown in Table 1.

The length of each link and initial phase angles of two cranks are used as optimization variables. Given that the constant $C$ should be set as $-400 \mathrm{~mm}$ in (13), the constraint of initial shearing angle is selected as follows: $\alpha_{\text {st }} \geq 1.5^{\circ}$. Meanwhile, the optimization model of rolling shear mentioned above can be established, together with the genetic optimization algorithm employed. Therefore, the lengths of linkages, the coordinate of fixed hinge point $F$, and the initial phase angle of crank $A B$ of the new mechanism can be obtained, as shown in Table 2.

4.2. Kinematic Performance Analysis. A major kinematic performance concerned for this design is a pure-rolling motion between two blades and is described by deviations between fixed and moving centrodes and contacting lines, which is intuitively exhibited through the trajectory of the lowest moving point and arc middle point of upper shear blade. The cutting performance is illustrated by the comparison of the shear angle and shear stress between the original design and the new design in this paper.
TABLE 2: The design result of the new rolling shear mechanism.

\begin{tabular}{lc}
\hline Result parameters & Value \\
\hline Length of link $A B$ & $114.9 \mathrm{~mm}$ \\
Length of link $E F$ & $114.9 \mathrm{~mm}$ \\
Length of link $B C$ & $864.1 \mathrm{~mm}$ \\
Length of link $E D$ & $864.1 \mathrm{~mm}$ \\
Length of $A F$ & $2400.0 \mathrm{~mm}$ \\
Length of link $C D$ & $2400.0 \mathrm{~mm}$ \\
Length of link $D G$ & $856.7 \mathrm{~mm}$ \\
Length of link $H G$ & $807.2 \mathrm{~mm}$ \\
Coordinates of fixed hinge point $F$ & $(-1638.1,1033.0)$ \\
Initial phase angle of crank $A B$ & $114.0^{\circ}$ \\
\hline
\end{tabular}

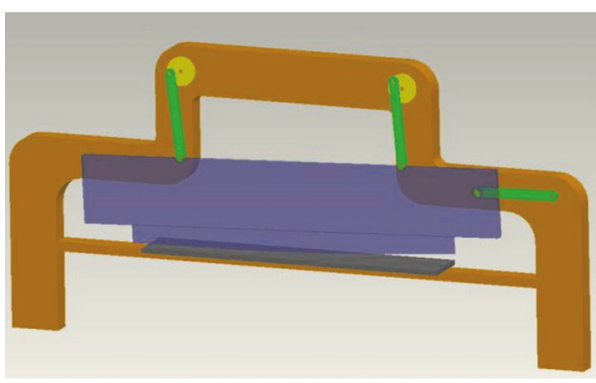

FIGURE 8: Motion simulation of rolling shear mechanism.

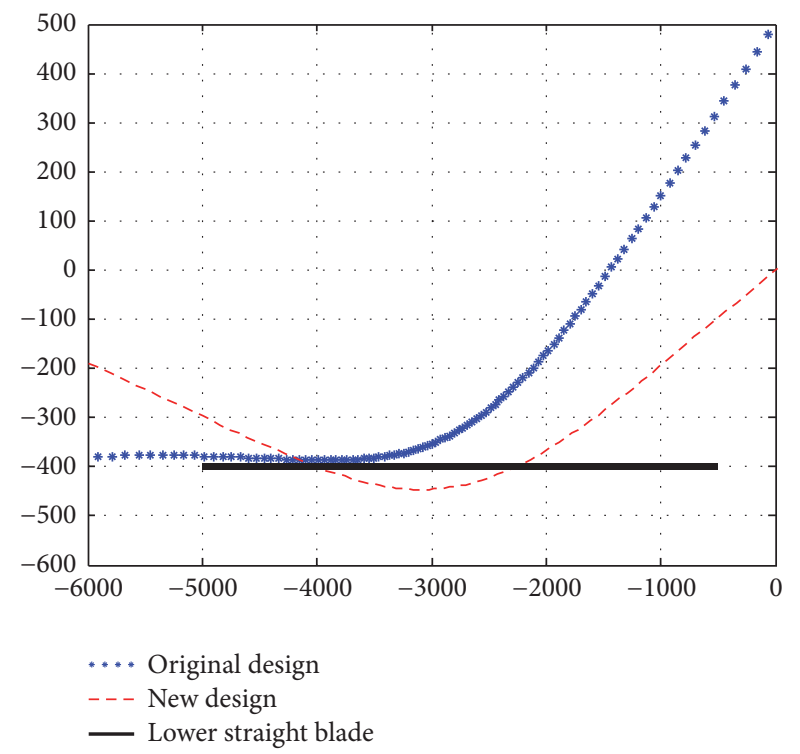

FIGURE 9: Fixed centrode of upper shear blade and lower horizontal shear blade.

Simulation and performance analysis of the rolling shear mechanism based on Pro/E and MATLAB software were conducted. Figure 8 shows the motion simulation model of the rolling shear mechanism.

The comparison of the fixed centrode of the upper shear blade and the lower shear blade between the original and optimal results is shown in Figure 9. The designed fixed 


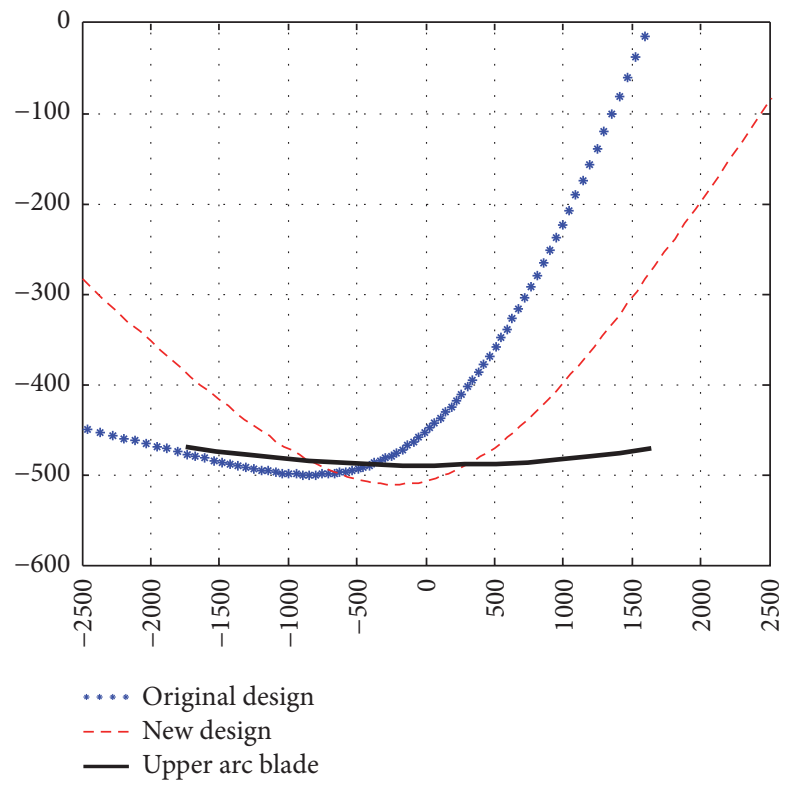

FIGURE 10: Moving centrode of upper shear blade and arc profile of upper shear blade.

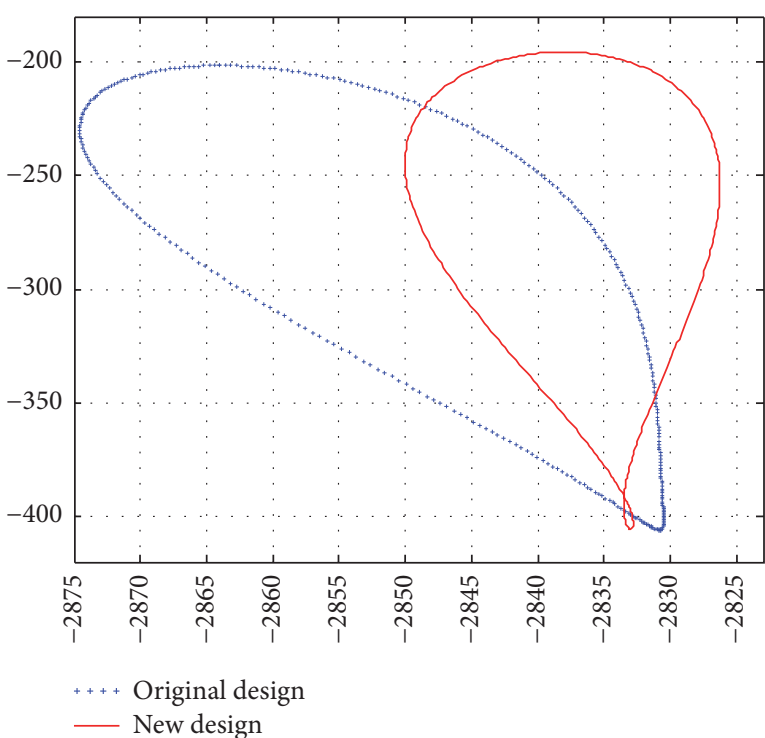

(a) Overall view

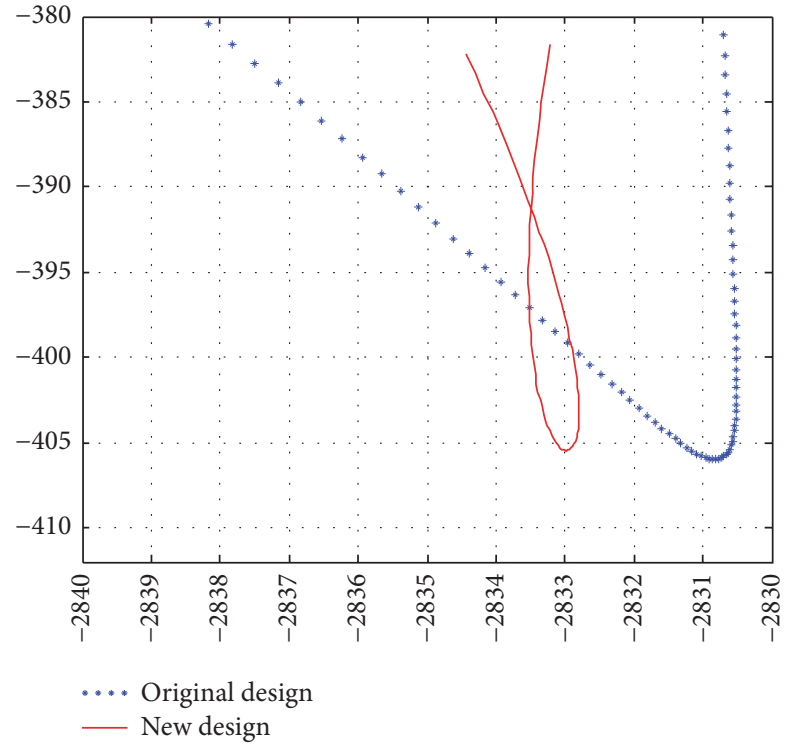

(b) Trajectory in the shearing process

FIGURE 11: Trajectory of arc middle point on the upper shear blade.

centrode has better straightness in the segment, which can approximate the horizontal contact line in a better way and is in accordance with the objective function. Notice that the axes are not isometric for clear demonstration.

Figure 10 shows that the designed moving centrode approximates the symmetrical arc perfectly, which means that it approximates the moving contact arc perfectly, which is in accordance with the objective function. Notice that the axes are not isometric for clear demonstration.

Figure 11 shows the trajectory of arc middle point on the upper shear blade, which presents the cutting process part.
Notice that the axes are not isometric for clear demonstration. The results demonstrate that the horizontal slipping of the designed upper shear blade is confirmed as $0.97 \mathrm{~mm}$, compared to the original result of $4.88 \mathrm{~mm}$, reduced by $80.1 \%$, which illustrates that the designed upper shear blade profile is better in the realization of pure-rolling motion and also indirectly proves the validity of the method of designing rolling shear mechanism sizes.

Figure 12 shows that the trajectory of the lowest moving point of upper shear blade is approximately a straight line, and its straightness reflects overlapping evenness of upper 


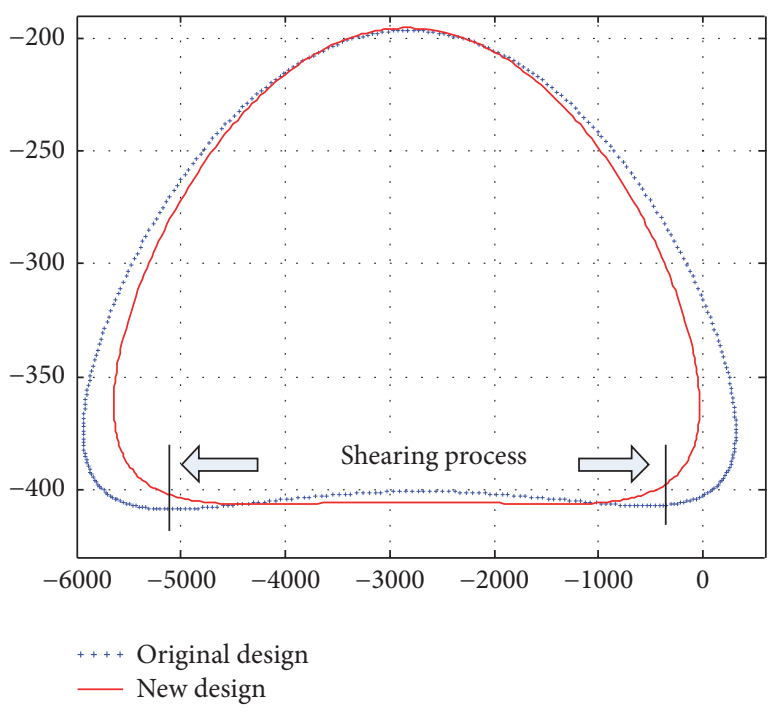

(a) Overall view

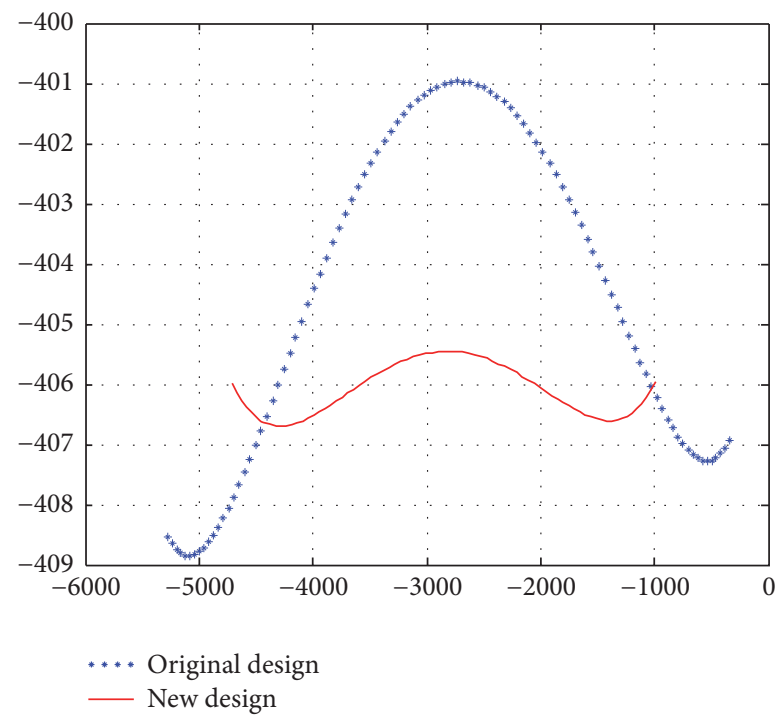

(b) Trajectory in the shearing process

Figure 12: Trajectory of the lowest moving point $W$ on upper shear blade.

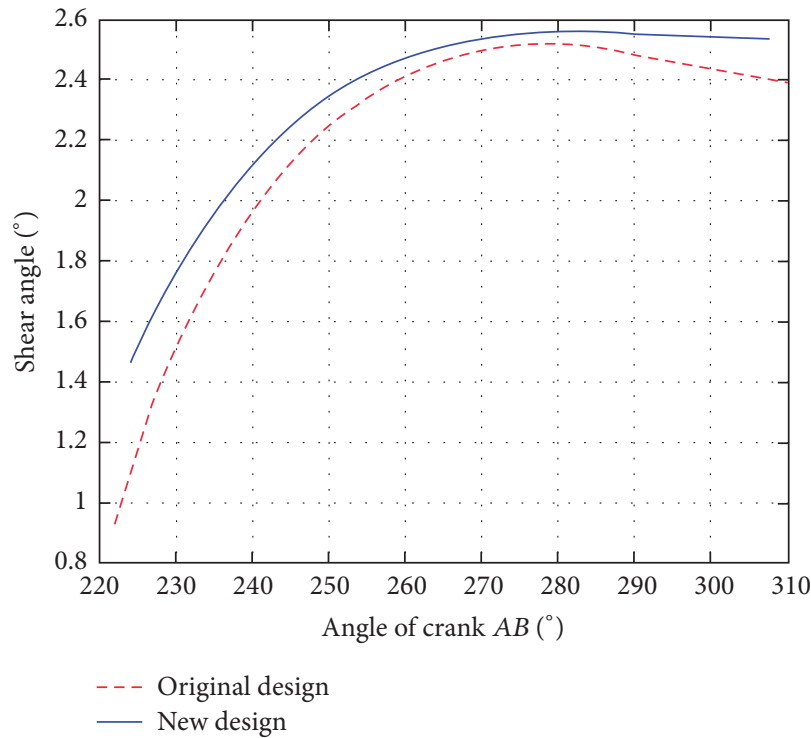

(a) Shear angle

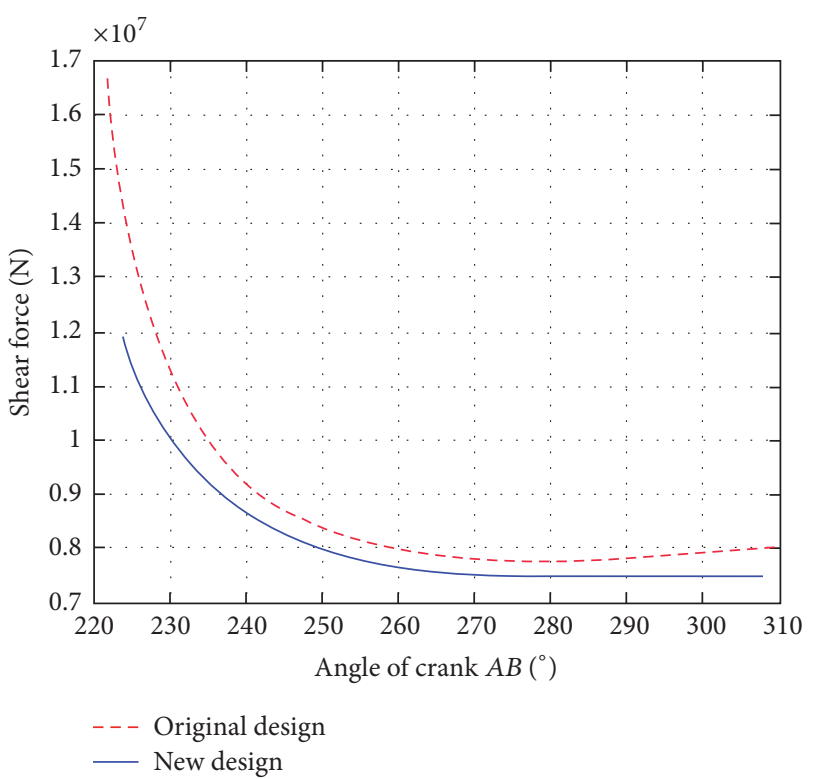

(b) Shear force

Figure 13: Comparison of shear angle and stress before and after the design.

and lower shear blade. Notice that the axes are not isometric for clear demonstration. The standard deviation of optimal result in trajectory sets of upper arc lowest moving point during shearing process is confirmed as $0.415 \mathrm{~mm}$, compared to the original result of $1.890 \mathrm{~mm}$, being reduced by $78.0 \%$, indicating more uniform overlap between upper and lower shear blade.

The changes of shear angle and stress before and after the design are shown in Figure 13, which indicates that the initial angle of the designed rolling shear mechanism at the beginning of the cutting process is roughly $1.5^{\circ}$, while the original initial angle is $0.9^{\circ}$. This improvement will be of great interest to improving the initial peak value of shear force. The shear angle increases to about $2.2^{\circ}$ when the shearing process comes to the stable rolling stage, no matter in the original design or in the new design. The peak value of shear stress of the designed rolling shear mechanism is roughly $1.2 \times 10^{7} \mathrm{~N}$, decreasing by $29 \%$ in comparison with the original shear stress peak of $1.7 \times 10^{7} \mathrm{~N}$. Moreover, the above figures, along with shear angle changing curve, show that the shear stress 
and shear angle change oppositely. Therefore, it is beneficial to improve the initial shear angle in order to reduce the initial shear stress.

\section{Conclusions}

A new approach to design a seven-bar linkage for purerolling cutting by optimizing centrodes is presented in this paper. Using the genetic optimization algorithm, the proposed method allows the designer to obtain an optimum linkage which minimizes the error between the centrodes of mechanisms and profiles of pure rolling. With the proposed method, a seven-bar rolling shear mechanism is designed which has better performance compared to the original one in the following aspects:

(1) The horizontal slipping of the designed rolling shear mechanism has been reduced by $78.0 \%$, which increases the cutting efficiency and reduces the wear of the shearing blade.

(2) The standard deviation of the lowest moving point on the upper shear blade has been reduced by $80.1 \%$, which indicates better quality of steel plates.

(3) The peak value of shear stress, which indicates the power performance of rolling shear mechanism, is decreased by $29 \%$ for long service life.

The proposed method in this paper is used for the seven-bar pure-rolling cutting mechanism. It can also be applicable to the other types of pure-rolling mechanism. In this paper, the genetic optimization algorithm method is adopted for obtaining an optimal solution, and other optimization methods can be considered to search for better optimal solutions in future study.

\section{Conflicts of Interest}

The authors declare that there are no conflicts of interest regarding the publication of this paper.

\section{Acknowledgments}

The authors acknowledge with appreciation the financial support from the National Natural Science Foundation of China (Grant no. 51275067).

\section{References}

[1] G. Figliolini and J. Angeles, "Synthesis of conjugate Geneva mechanisms with curved slots," Mechanism and Machine Theory, vol. 37, no. 10, pp. 1043-1061, 2002.

[2] L.-M. Wu, J. Ren, and T.-B. Yin, "Optimal design of rolling shear mechanism based on improved genetic algorithms," in Proceedings of the International Conference on Digital Manufacturing and Automation, (ICDMA '10), pp. 68-71, chn, December 2010.

[3] J. Wang, T. Hashimoto, H. Nishikawa, and M. Kaneta, "Pure rolling elastohydrodynamic lubrication of short stroke reciprocating motion," Tribology International, vol. 38, no. 11-12, pp. 1013-1021, 2005.

[4] P. H. Pan and R. M. Qian, "Design of the new pure rolling circular arc gear," Jiangsu Machine Building Automation, no. 1, pp. 20-22, 2003.
[5] Y. S. Shen, Theory of machines and mechanisms, Tsinghua University Press, Beijing, China, 2nd edition, 2005.

[6] S. Bai, D. Wang, and H. Dong, "A unified formulation for dimensional synthesis of Stephenson linkages," Journal of Mechanisms and Robotics, vol. 8, no. 4, Article ID 041009, 2016.

[7] Y. Liu and W. B. Xiao, "Progress in research on the theory of mechanisms for path generation," Journal of Computer-Aided Design and Computer Graphics, vol. 17, no. 4, pp. 627-636, 2005.

[8] J. A. Cabrera, A. Simon, and M. Prado, "Optimal synthesis of mechanisms with genetic algorithms," Mechanism and Machine Theory, vol. 37, no. 10, pp. 1165-1177, 2002.

[9] I. Uiiah and S. Kota, "Optimal synthesis of mechanisms for path generation using fourier descriptors and global search methods," Journal of Mechanical Design, vol. 119, no. 10, pp. 504510, 1997.

[10] Z. Li and C. L. Gui, "Morphologically Analyzing method for the characteristic of coupler curves of linkage mechanism," Chinese Journal of Mechanical Engineering, vol. 38, no. 2, pp. 27-30, 2002.

[11] W. Q. Cao, "The identification of coupler curve by using fuzzy mathematics," Journal of Shanxi Institute of Mechanical Engineering, no. 2, pp. 21-28, 1992.

[12] X. B. Sun, R. B. Xiao, and X. Wang, "An approach to mechanical path generation and retrieve based on quantification and extraction of the geometrical features of curve," Chinese Journal of Mechanical Engineering, vol. 36, no. 11, pp. 98-105, 2000.

[13] G. Nelson Larsen, Analysis of the four-bar linkage, Published jointly by the Technology Press of the Massachusetts Institute of Technology, and Wiley, New York, NY, USA, 1951.

[14] S. N. Kramer, "Selective precision synthesis of the four-bar motion generator with prescribed input timing," Journal of Mechanical Design, vol. 101, no. 11, pp. 614-618, 1979.

[15] T. Subbian and D. R. Flugrad, "Four-bar path generation synthesis by a continuation method," Journal of Mechanical Design, vol. 113, no. 1, pp. 63-69, 1991.

[16] D. S. Johnson, C. R. Aragon, L. A. McGeoch, and C. Schevon, "Optimization by simulated annealing: an experimental evaluation-Part II: graph coloring and number partitioning," Operations Research, vol. 39, no. 3, pp. 378-406, 1991.

[17] S. Kirkpatrick, C. D. Gelatt Jr., and M. P. Vecchi, "Optimization by simulated annealing," American Association for the Advancement of Science, vol. 220, no. 4598, pp. 671-680, 1983.

[18] J. R. Wang and X. Q. Huang, "Optimization of the shaft of a rolling shear with single shaft and double eccentricity," Heavy Machinery, no. 1, 2005.

[19] J. G. Yang, Z. W. Yue, and H. C. Zhang, "Optimization of shear mechanism in plate shear with roll guide groove," Journal of Taiyuan University of Science \& Technology, vol. 24, no. 4, pp. 297-300, 2006.

[20] S. F. Sun, X. H. Yang, Q. X. Yan, and D. Y. Yang, "Simulation research of mechanism parameter on cut-to-length rolling shear," Metallurgical Equipment, no. 170, 2008.

[21] M. A. Li-Feng, Q. X. Huang, L. I. Ying et al., "Establishment and application of mathematical model on spatial shear mechanism optimization of new-type steel rolling shear," Journal of Sichuan University, no. 2, pp. 170-174, 2008.

[22] Q. Huang, L. Ma, J. Li, J. Wang, Q. Zhang, and C. Zhao, "Principle of asymmetric crank mechanism of new-type rolling shear," Chinese Journal of Mechanical Engineering, vol. 44, no. 5, pp. 119-123, 2008.

[23] L. J. Meng and Y. W. Chen, "Application of offset mechanism of crankshaft in rolling-type heavy steel shear," Journal of Taiyuan University of Science \& Technology, 2009. 
[24] D. L. Wang and Y. Gao, Mechanical Principle, China Machine Press, Beijing, China, 2011.

[25] H. X. Lin, W. Huang, T. X. Lin et al., "Study of synthetically genetics annealing optimization for the luffing mechanism trajectory of a plane link," Journal of Computer-Aided Design \& Computer Graphics, vol. 13, no. 8, pp. 724-729, 2001.

[26] X. P. Zhang, G. J. Yang, and J. F. Wang, "Analysis of factors influencing cutting quality and strength parameters of rolling cut shears," China Mechanical Engineering, no. 7, pp. 780-784, 2008.

[27] S. L. Li, X. L. Qin, and G. B. Zhao, "Rolling shear characteristics and analysis of key parameters," Industrial Technology, no. 24, 2008.

[28] J. M. Wen and F. Wang, "Shear force calculation of the rolling shear mechanism," Metallurgical Equipment, no. 1, 2011. 


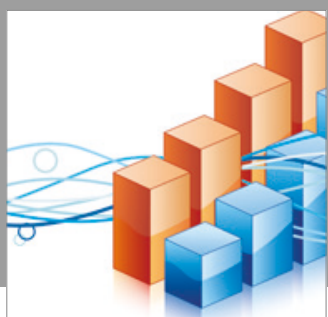

Advances in

Operations Research

vatersals

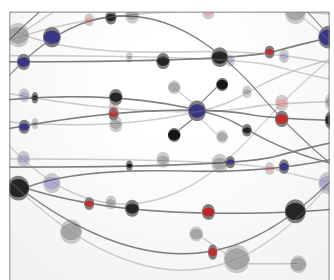

\section{The Scientific} World Journal
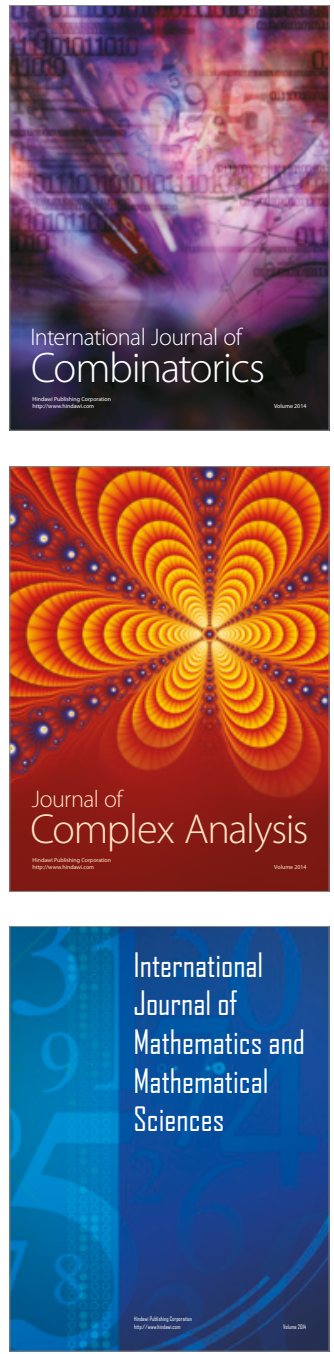
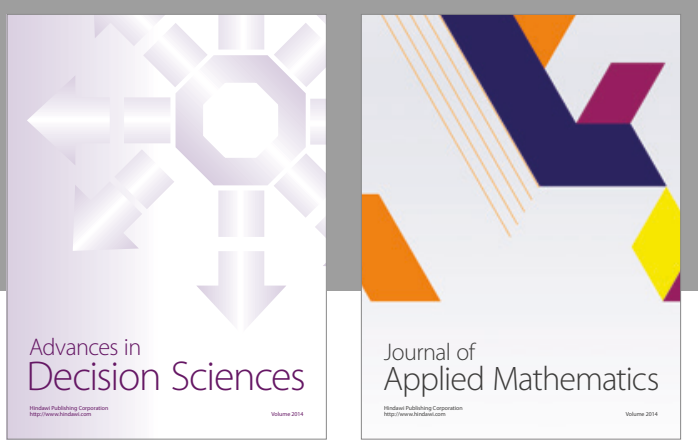

Algebra

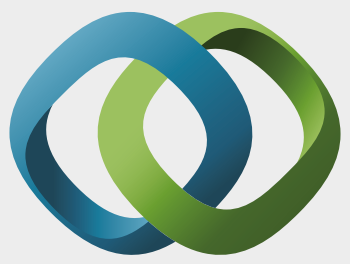

\section{Hindawi}

Submit your manuscripts at

https://www.hindawi.com
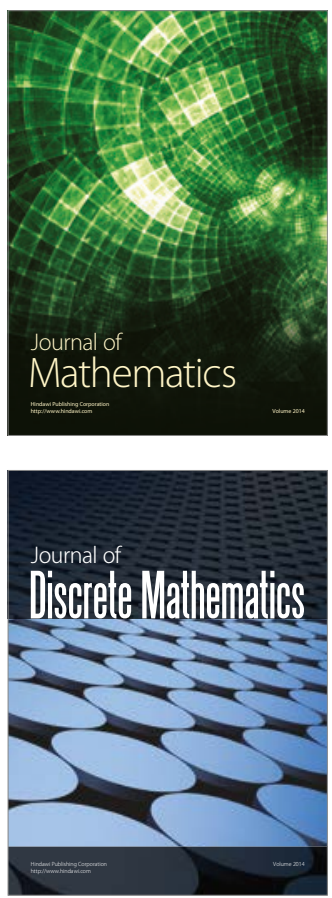

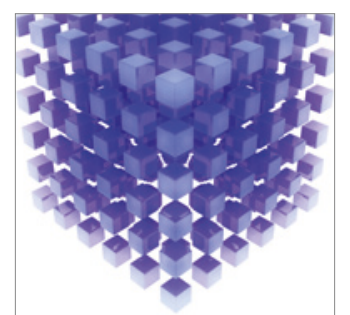

Mathematical Problems in Engineering
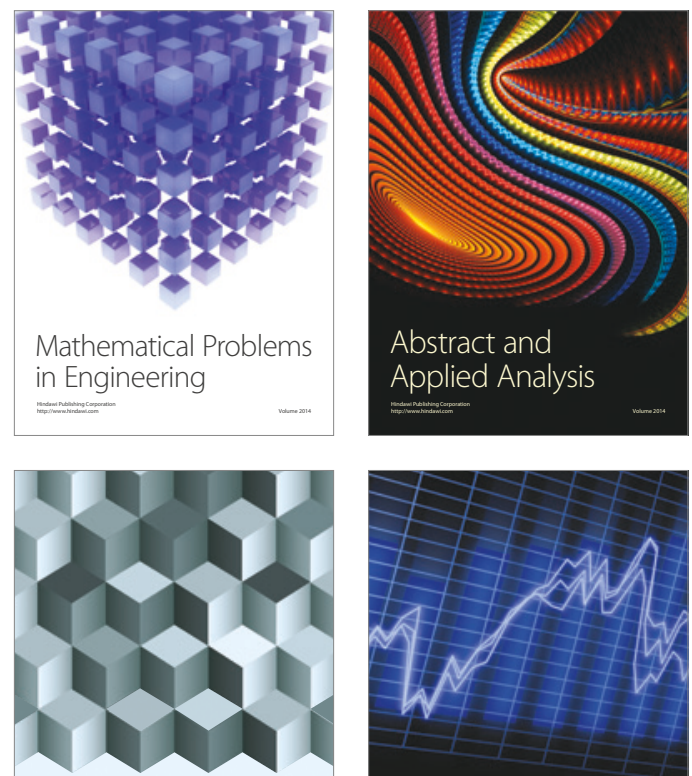

Journal of

Function Spaces

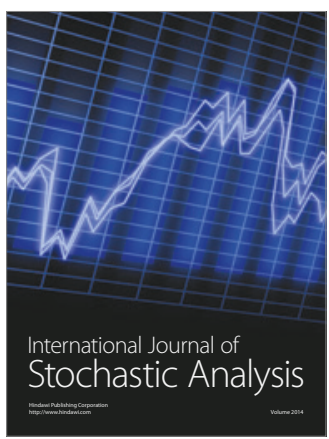

Probability and Statistics
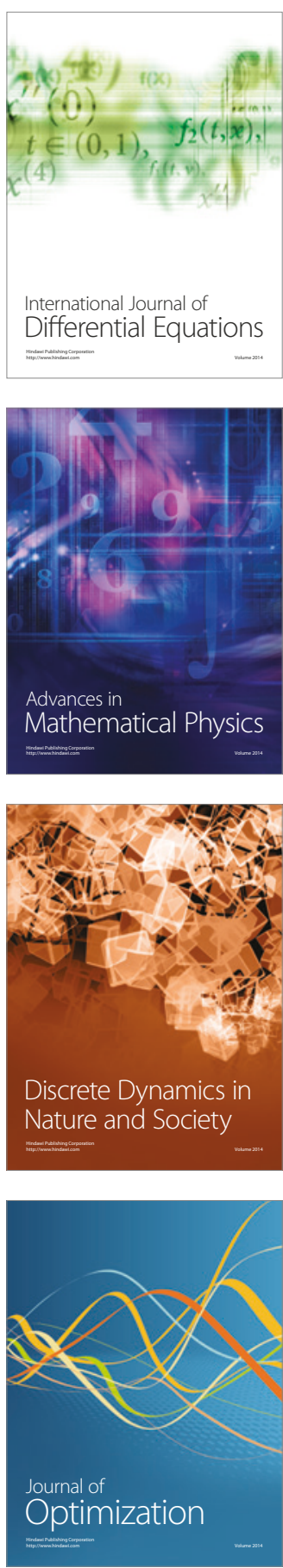